\title{
Are Functional Measures Sufficient to Capture Acceptance? A Qualitative Study on Lower Limb Exoskeleton Use for Older People
}

\author{
Maria del Rio Carral ${ }^{1}\left[\right.$ [D $\cdot$ Vanlisa Bourqui ${ }^{1} \cdot$ Noémie Vuilleumier $^{1} \cdot$ Amalric Ortieb $^{2} \cdot$ Mohamed Bouri $^{2,3}$
}

Accepted: 25 June 2021 / Published online: 23 July 2021

(c) The Author(s) 2021

\begin{abstract}
Lower limb exoskeletons (LLE) are robotic devices developed to assist walk. In the field of healthcare, this technology has been available for almost a decade, yet it still faces important acceptance issues. While LLE were first developed for patients with spinal cord injuries, we expect their use to expand to everyday settings to benefit other populations, namely that of older people with reduced mobility (RM). We propose a qualitative approach to unearth key psychosocial themes on the acceptance of LLE in daily living for older people. The study analyses perceptions of older people with RM, as well as their entourage, including informal and professional caregivers. Using a grounded theory approach we analysed 12 semi-structured interviews with older people with RM; 2 focus groups with informal caregivers, and 2 focus groups with professional caregivers. LLE were introduced to participants through photo-elicitation. Older people with RM believed that LLE would increase their autonomy. They also perceived that using LLE would make them feel less of a burden for their entourage. Beyond these expected benefits, results captured participants' ambivalence, dependent on their experiences of the ageing process and perceptions on the human-machine interaction. Informal caregivers highlighted that LLE could provide important relief related to the burden of care. Nonetheless, professional caregivers raised the fear of LLE leading to dehumanization of care. While each group had specific concerns on how LLE use would impact their lives, psychosocial considerations played a key role in LLE acceptance.
\end{abstract}

Keywords Lower-limb exoskeleton · Older people with reduced mobility · Ageing · Assistive technology · Qualitative methods

\section{Introduction}

Ageing constitutes a complex process involving social, physical and cognitive changes, as well as psychosocial transformations [1-3]. Older peoples' evolving identities are characterised by unprecedented meanings about life, the body and the self, associated to physical, psychological and social transformations. Such lived experience is defined by their

Maria del Rio Carral

maria.delriocarral@unil.ch

1 Institut de Psychologie, Université de Lausanne, Mouline-Géopolis, 1015 Lausanne, Switzerland

2 Biorobotics Laboratory (BIOROB), School of Engineering, Ecole Polytechnique Fédérale de Lausanne (EPFL),

Lausanne, Switzerland

3 Translationnal Neural Engineering Laboratory (TNE), School of Engineering, Swiss Federal Institute of Technology (EPFL), Geneva, Switzerland participation to new everyday life environments [4-6], such as medical settings or nursing residencies. In contemporary Western societies, addressing older populations' health and care needs constitutes a major challenge linked to a high average life expectancy which is still anticipated to increase by five years between 2005 and 2050 [7, 8].

A major psychosocial implication of ageing is the loss of autonomy caused by the decline of physical mobility. Older people are subject to rapid mobility deterioration. For instance, being bedridden (whether from illness or accident) can lead to a loss of muscle mass and strength (up to 5\% per day). [9] Mobility deterioration can also happen after a stroke which has a high prevalence of $18 \%$ in the $55+$ population [10]. To fully recover, older people are led to work on their muscular strength, often with the help of professional and/or informal caregivers, both at home and in rehabilitation settings. This physical decline inevitably affects ways in which everyday activities are carried out. Also, this physical decline can be an onset for an older person having to move 
from the private household to a nursing residence. Hence, challenges related to ageing involve not only the older person as an individual, but also their entourage and healthcare systems more broadly.

Within this context, assistive technologies (AT) have massively expanded to address some of the complex challenges related to ageing. Particularly, powered lower limb exoskeletons (LLE) are robotic-like devices that assist people to walk. They constitute a promising solution that can be targeted at increasing physical mobility among older populations. These tools have been designed to assist with walking through a partial effort contribution or by mimicking as much as possible the human gait (Fig. 1). Recently, there has been a growing interest towards the potential of LLE in the field of gerontology, as these tools could contribute to enhance autonomy among older people and, in doing so, improve their quality of life [11].

Today we witness a recent growing interest in the literature with regard to the acceptance of LLE for everyday use among older populations in Western societies [14]. From a user-centred perspective, Shore and her colleagues $[15,16]$

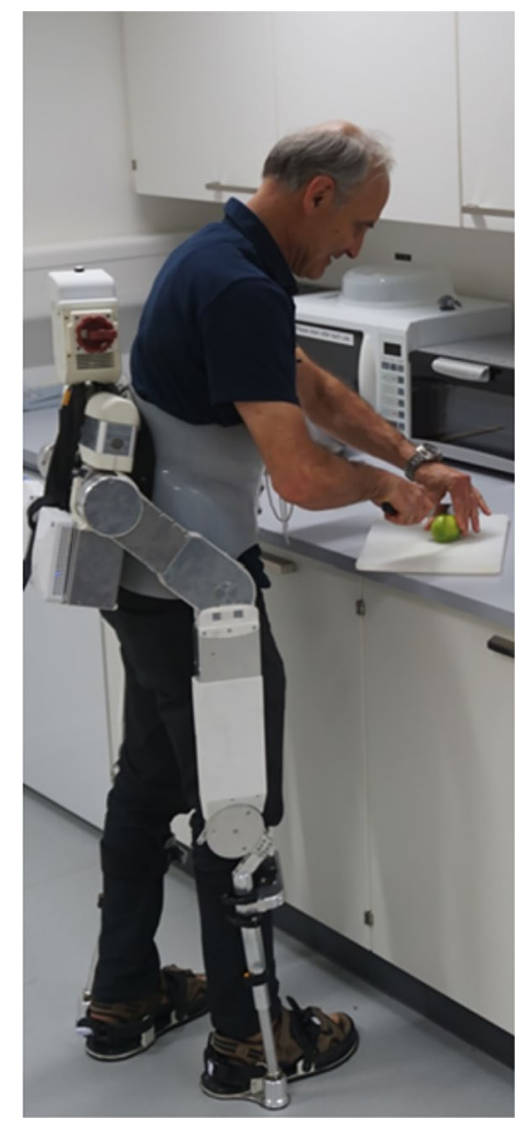

Fig. 1 Illustration of a powered lower limb exoskeleton actuated at the level of the hip and knee joints. It assists the abduction/abduction and flexion/extension of the hip joint, and the flexion/extension of the knee joint (LLE Autonomyo, [12, 13]) have explored LLE acceptance in certain European contexts. This work adopts qualitative methods to explore the perceptions of senior adults (primary users) and caregivers (secondary users) regarding the potential of LLE for social and domestic settings. Their approach is inspired by the Technology Acceptance Model applied to older populations (Senior Technology Acceptance Model, STAM) [15]. This line of research has pointed out an array of functional requirements to be prioritized for exoskeleton design for older adult populations. Their research shows that exoskeletons need to include a specific set of functionalities to be better accepted by older people. More specifically, they need to be effective, feel safe, facilitate walking, allow for hands-free usage, support their user's body appropriately, be easy to wear, and be low cost [16]. This array of features corresponds to practical functionalities. Yet, there is scant research that describes the psychosocial dimension of LLE acceptance among older people and their caregivers. For instance, a recent study using qualitative methods highlighted the importance of perceived usefulness and trust of the technology [15]. However, additional research that adopts a qualitative perspective is further needed. In the fields of psychology and health, qualitative methods have a long tradition in the understanding of human experience by examining meanings that people attach to the world, others, and themselves through language [17]. Beyond its potential to analyse complex phenomena [18], qualitative research is moreover well-suited to produce knowledge from the recipient's perspective and to move towards recommendations that consider the complexity of health and illness experiences [19]. In this sense, it seems particularly appropriate to focus on the qualitative, thus, indepth understanding of older people and their caregivers' own experiences of the ageing process, the care relationship, and the projections they may have on LLE and technology. This psychosocial focus deserves more attention to assess the potential of LLE for autonomy enhancement specifically among older people in everyday life contexts [20].

The present qualitative study will contribute to fill in this gap in research. It aims to gain a deeper understanding on the perceptions that older people and their caregivers attach to LLE by referring to their lived experiences, everyday activities, and personal needs. To achieve this aim, we conduct an interdisciplinary study involving health psychology and robotics: Health psychology provides a qualitative perspective to analyse how individuals concerned by reduced mobility and ageing perceive LLE, based on their lived experience [20]. The field of robotics will provide a specific prototype of LLE that is currently developed. Our study will consider three different perspectives: those of older people with reduced mobility (RM) as well as the ones of their informal and professional caregivers respectively. The study focuses on the situations of older people who live in nursing 
residencies or at home, and who require special assistance to carry out everyday activities.

\section{Current Developments}

Assistive technology (AT) is receiving attention because of their capacity to improve the quality of life among vulnerable populations with motor deficits, amputated limbs, hearing or visual impairments. AT primarily provides help to perform activities of daily living (ADLs). The emergence of robotics plays a key role in the current trends to extend AT with personal robots, powered prosthetics, knee or ankle joint exoskeletons, motorized wheelchairs, and powered exoskeletons [21-24].

Powered LLE, which we address in the current study, are wearable devices rigidly interfaced with the user's legs to assist walking, through a partial effort contribution, or by mimicking as much as possible the human gait. To assist people with impaired mobility, LLE can adopt two different approaches: mobilization or assistance. Mobilization strategies are adapted for largely impaired patients such as complete spinal cord injury people where the exoskeleton is providing full motion based on programmed trajectories. Assistance strategies give more freedom to the user by providing additional force to the user based on the sensed motion. The latter are well-suited for mildly impaired patients suffering from neuromuscular disease or following a stroke [25]. To date, research on LLE has often focused on the needs of individuals suffering from complete spinal cord injury $[26,27]$. Their use in rehabilitation seems to have positive effects not only on a physical level, but also on a psychosocial level such as the quality of life. Also, daily social interactions and everyday activities tend to be facilitated through a verticalized and upright body posture [28].

Recently, the potential of LLE for older people has been highlighted [11, 25, 29, 30]. Ageing, and strokes, involves increased health risks, among which one is the reduction of physical mobility. While mobility tends to deteriorate under ageing circumstances, the capacity to perform daily tasks and social interactions becomes increasingly difficult as well. Under such circumstances, LLE constitute promising AT solution for everyday uses as they could improve the autonomy for the older end-user. Social and psychological well-being among senior populations could also benefit through social reengagement and psychological empowerment [31]. Ultimately, these changes in everyday life could also potentially be positively perceived from the perspective of professional and informal caregivers who are involved in addressing seniors' health and care needs in the day-to-day. At the same time, due to their 'futuristic' appearance, LLE could be stressful to senior end-users. The digital divide seems to contribute to explain this potential barrier [16].
More 'traditional' already existing AT, such as canes and wheelchairs, could be preferred instead [15].

Although exoskeletons have been proven effective in improving the degree of autonomy among users, these assistive devices can also be seen as physically burdensome especially when the users encounter difficulties in mastering such technology [32-34]. In fact, LLE may cause unprecedented ways of experiencing the body by introducing a triangular relationship between the end-user, the device, and the environment. This triangular relationship is crucial in developing acceptance of LLE by their users. Indeed, researchers have mainly emphasized the importance of helping patients via AT to 'move as a human' more than 'move like a human' [32].

Based on the potential benefits and barriers of LLE acceptance and use, it seems imperative to better understand the ways in which older people and their entourage experience and embody, from their own perspective, the ageing process, and the reduction of physical mobility. For instance, certain individuals might consider AT as a reminder of their disability and, consequently, they would rather 'live' with their disability than acknowledge their need for technological assistance [31]. Thus, the acceptance of LLE as an AT appears to be a complex process where subjective views on health and care needs are closely intertwined with perceptions on ageing, the care relationship, and the technology. Moreover, these perceptions are inevitably rooted in personal experiences, which are always contextualised [20,35]. For example, individualised postmodern Western societies tend to highly value autonomy, as well as personal agency. These values could orient LLE acceptance as they influence the ways in which ageing is perceived among senior adults, especially regarding a loss of autonomy.

\section{An Interdisciplinary Qualitative Approach}

Recent studies have underlined the need to involve endusers in the process of analysing LLE acceptance, especially within the field of gerontology $[15,16]$. Indeed, knowledge regarding older people's perceptions-as well as that of their entourage - of ageing and their experience of the care relationship are both crucial to better understand the psychosocial benefits and potential challenges of LLE use.

Qualitative research in health psychology has the potential to provide nuanced knowledge, based on people's own perspective [18]. This form of research was adapted to conduct an exploratory study following an interdisciplinary collaboration with robotic engineers. Indeed, a specific LLE is being developed for potential users with neuromuscular weaknesses by the research group REHAssist of the Swiss Federal Institute of Technology (EPFL). This device, called AUTONOMYO [12,13], is innovative given its light weight 
and character, which echoes the structure and dimensions of human lower limbs. Also, AUTONOMYO's originality relies on its potential to reproduce human movements to assist with walking. Its design and control strategies aim at enabling users to experience better body balance compared to other exoskeletons currently being produced. The similarity of AUTONOMYO's movement with the dynamics involved in human walk seem promising to allow greater mobility among users, since it does not require any crutches or canes. AUTONOMYO can interact with human lower limbs while imitating their natural movements. This gain in autonomy could be highly useful to aid older people with ADLs. However, AUTONOMYO cannot reach its full potential unless its construction considers the perspectives of its intended end-users. Beyond the mechanics and functioning of the device, we strongly believe that technology must meet people's psychosocial views and needs, within specific contexts of everyday life, based on the end-user's experience.

Given these arguments, the collaboration between robotic engineers and health psychologists was necessary for the further understanding of the psychosocial aspects involved in the acceptance of LLE among older people. In addition, this collaboration is useful to study in which environments are the use of LLE most appropriate to enhance autonomy and well-being in daily life.

\section{Methodology}

In our study, we adopted a qualitative methodology, specifically using a grounded-theory framework [36, 37]. This framework is widely acknowledged in the domains of psychology and health to explore how people make sense of their experience, based on meanings that they attach through language to their everyday life and experience [38-40]. In fact, qualitative data are not aimed at testing causal hypothesis, measuring the influence of psychological variables, nor building predictive models. Rather, qualitative data have the potential to provide an in-depth understanding of a given phenomenon as experienced by a certain group of individuals [41].

\subsection{Population and Ethical Issues}

The current research, funded by a Swiss programme, took place in the French-speaking part of Switzerland. All participants were recruited by the research team conducting this study through medical practices of the physiotherapists and the occupational therapists, domestic caregiver centres, daycare centres, retirement homes, different geriatric associations and, finally, using social networks. The research protocol was approved by the competent human research ethics committee (reference EPFL, HREC-004-2018). Particular attention was taken on the handling of any sensitive data, typically the details related to the mobility condition of the older participants. All participants were informed of the details of the study before data collection and informed consent was obtained. Three populations were recruited:

1. Home dwelling older people with RM $(n=12)$ both male and female ( 8 male and 4 female) aged from 64 to 80 years-old (mean age $=70.9$ ), from different socioeconomic backgrounds and currently living in the French-speaking part of Switzerland and capable of discernment. Individuals with paraplegia were excluded from this study.

2. Informal caregivers $(n=7)$, both male and female (2 male and 5 female, mean age $=58.14$ ), who assisted or accompanied on a regular basis an older person with RM residing at home $(n=4)$ or a residency environment $(n=3)$; from different socio-economic backgrounds and currently residing in the French-speaking part of Switzerland and able to speak fluent French. Individuals having personal contact with the older population who were selected for this study were excluded.

3. Professional caregivers $(n=9)$, both male and female (3 male and 6 female) holding a certification of nursing science, healthcare assistance and community health, or personal care assistance (mean age $=41.44$ ); assisting or accompanying at least 3 times per week one or more older persons with RM residing at a household $(n=4)$ or nursing home environment $(\mathrm{n}=5)$. Individuals having personal contact with the older population selected for this study were excluded.

\subsection{Data Collection}

The collection of data involved a qualitative methodology aimed at producing narratives among primary and secondary potential end-users of LLE, using through three different methods: semi-structured interviews [42], focus groups [43] and photo elicitation [44]. Given ethical and security reasons, the LLE was not actuated nor worn by any of the participants.

Twelve semi-structured interviews with older people with RM; 2 focus groups with 3-4 informal caregivers and 2 focus groups with 4-5 professional caregivers were conducted by the researchers, namely the health psychologists. The interview and focus group guides were loosely structured, using photo-elicitation to orient the discussion, as to allow participants to elaborate freely around their own perceptions and experiences. The method of photo elicitation [44] therefore served as a complementary technique to stimulate the production of narratives. Images below (Fig. 2) were taken by the research team, namely by the robotic engineers. These images portrayed AUTONOMYO being 

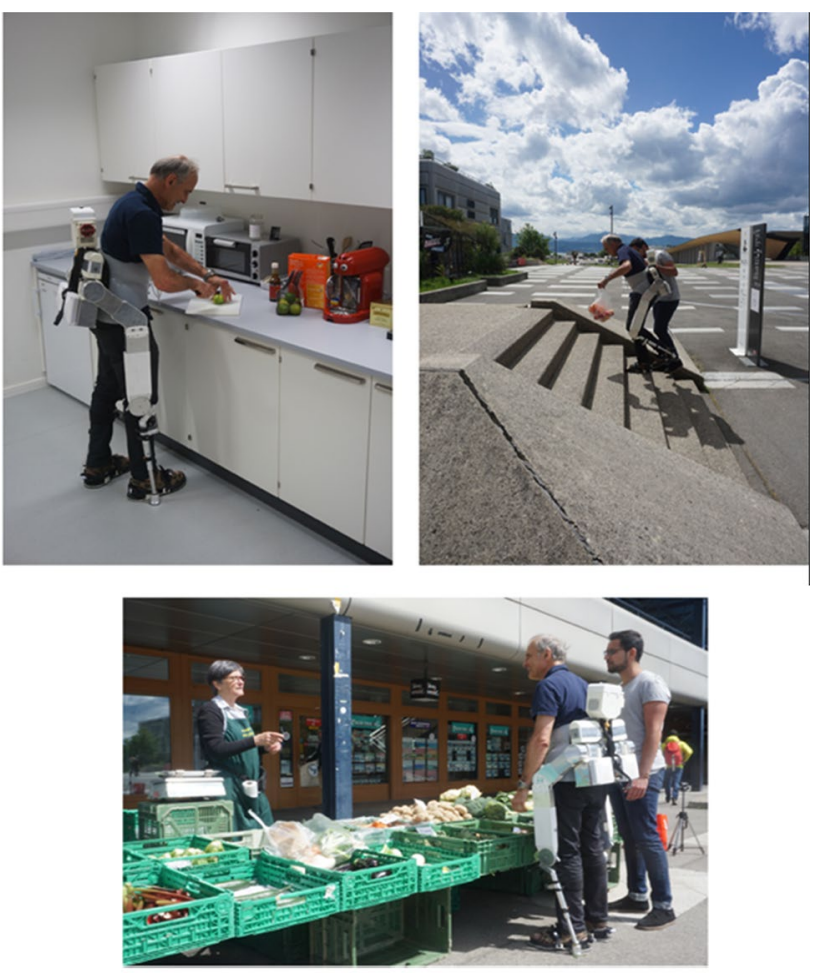

Fig. 2 Example of images used for the photo elicitation method

worn by a senior adult (the chief of the robotics engineers' team) illustrating situations involving ADLs (e.g. shopping for food, doing a leisure activity, walking on a sloped road, climbing stairs). Images were used in interviews and focus groups to stimulate contextualised narratives. That way, participants were able to visualise concrete LLE in relation to everyday life situations. Full accounts were recorded and transcribed by two of the health psychology researchers. All interviews and focus groups were conducted in French and were also transcribed in French. Any information potentially revealing the identity of the participants was anonymised and replaced with fictitious names.

\subsection{Data Analysis}

Individual interviews and the focus groups' discussions were fully transcribed by two health psychologists of our team. The analysis was conducted in French, the original language of the data, although quotes used to illustrate our findings have been translated to English.

Full transcripts were subject to inductive thematic analysis [45]. This is a widely acknowledged method of inductive, thus exploratory analysis in qualitative research in psychology. Unlike quantitative procedures, this type of analysis does not require the previous definition of hypothesis [35, 46]. Inductive thematic analysis was conducted across the data to identify common topics or themes approached by participants during the individual interviews and focus groups. This analysis allowed for a systematic procedure to classify all accounts into distinct themes, defined on the basis of their meaning, that is, semantic similarities and differences [46]. The analytical process was completed until a semantic coherence was reached regarding participants' accounts and our research aims. Each corpus - the interview transcriptions (older people with RM) and the focus groups transcriptions (informal and professional caregivers) -were analysed separately to assess each population's perspective.

\section{Main Findings}

Participants from all three populations: older people with $\mathrm{RM}$, professional, and informal caregivers talked about the potential of using LLE by emphasising psychological and social considerations. Rather than focusing on practical functionalities, all participants discussed ways in which they perceived LLE regarding ageing, the care relationship, and technology in terms of a human-machine interaction. The sets of themes described below in detail best captured participants' perspectives. We present findings stemming from (4.1) older people with RM, (4.2) informal caregivers, and (4.3) professional caregivers separately, to then define cross-cutting considerations.

\subsection{Older People with RM Perspectives}

Accounts by older people with RM on the acceptance of LLE were organized according to three main topics or themes:

(a) Autonomy in everyday life can be enhanced through LLE, yet its acceptance requires learning;

(b) contrasting views of the ageing process and,

(c) contrasting imaginaries on the human-machine interaction.

\subsubsection{Autonomy in Everyday Life can be Enhanced Through LLE, Yet Its Acceptance Requires Learning}

LLE were perceived by older people with RM as beneficial devices in the sense that they could contribute to enhance autonomy by facilitating walking. Interestingly, psychosocial advantages were raised. One of them concerned the possibility of feeling more independent regarding their caregivers, thus, to feel less of a 'burden' in carrying out everyday tasks. 
Interview 6: [...] precisely, I would feel less dependent. $(F, 74$ years-old, older person with $R M)$

This need for autonomy was explained in contrast to their present situation of dependency towards informal and professional caregivers, experienced as particularly difficult:

Interview 1: I would like to try anything that would make me less dependent, you see? It's really difficult to be dependent upon people, you know, very difficult! ( $F, 78$ years-old, older person with RM)

From older people's perspective, autonomy associated to LLE potential would open up new possibilities for everyday life. Namely, LLE use would increase their ability to decide and choose activities without depending on someone. From this view, exoskeletons were portrayed as a promising solution:

Interview 7: It's really great to be able to do again things in which we can decide on our own, to have a certain independence and some spontaneity with regard to our own decisions. (M, 67 years-old, older person with $\mathrm{RM}$ )

Other psychosocial advantages associated to the embodied structure of exoskeletons were underlined. Most participants pointed out the expected positive influence of LLE on bodily sensations associated to everyday life. Specifically, participants argued that LLE had the potential to improve their own overall balance. All participants were convinced that this benefit could contribute to decrease their constant feeling of tiredness:

Interview 9: Probably that [pointing at the exoskeleton in one of the images] would have an influence, I think I would feel less tired. (M, 73 years-old, older person with RM)

Beyond these psychosocial benefits, the integration of LLE in everyday life was perceived as a process requiring learning. Participants underlined that they would need time to become familiar with this tool to make the most of its use:

Interview 2: Maybe, at first, I would be afraid of using it and I would have to become familiar with it. But in the long run, I'm sure that it would help me. It's like with everything else, you have to get used to it, and then you can achieve spectacular results! (M, 69 years-old, older person with RM)

In summary, the first theme on older people with RM pointed out the many perceived benefits of LLE for daily life use beyond their functionalities. The psychosocial dimension of autonomy was linked to expectations that concerned embodied sensations but also an increase in personal wellbeing. LLE for everyday life use was said to require a learning process that participants from this group would be willing to engage in.

\subsubsection{Contrasting Views of the Ageing Process}

Older people with RM's perception of LLE was strongly linked with their own definition of ageing, based on their lived experience. Two different positions were identified across participants:

According to the first position, mobility impairments were considered part of the normal and progressive decline associated with ageing. Participants who related to this vision seemed more reluctant towards LLE, as the use of such tools seemed to counter the 'natural' evolution that they associated to the ageing process:

Interview 10: It's totally normal to grow old, it's normal to have increased difficulties to move around! That's part of life, that's the evolution that all of us will undergo more or less rapidly, so it's better to begin to accept it rather than trying to fight, and fight and push against the normal evolution. ( $F, 75$ years-old, older person with RM)

Participants adhering to the second position considered it beneficial and necessary to use AT to fight against the negative implications of ageing such as physical decline. LLE were portrayed as promising solutions in this context to counter mobility loss. From this view, participants were open to the idea of using LLE to help push the boundaries of ageing and in doing so, certain difficulties that were experienced:

Interview 3: As we become old, the 'machine' [referring to the human body] falls into pieces and we have increased difficulties to walk. But that's normal, that's ageing, that's the way it is [...] But if 'your device' [referring to the exoskeleton shown in the pictures that were presented by the researcher] can help me fight against a little bit of this, and by doing this, postpone the problems due to ageing, I am not against using it. I'd even say: yes please, I'd like to! $(F, 79$ years-old, older person with $R M)$

In summary, this second theme on older people with RM suggested a certain variance of LLE acceptance dependent of experiences of ageing. This variance is defined by two different positions or views. This finding points out that perceptions on LLE use rely on how the ageing process is experienced. 


\subsubsection{Contrasting Imaginaries of the Human-Machine Interaction}

Unanimously, older people with RM participating in our study perceived LLE as 'machines' able to provide help by contributing to increase autonomy. Within this widely shared vision, we identified two different imaginaries: According to the first one, older people with RM positioned themselves as people who trusted LLE; in the second one, LLE raised certain scepticism, linked to broader imaginaries on the human-machine interaction.

According to the first imaginary, exoskeletons were seen as well-made reliable devices. This view was underpinned by the perception that it was easier to receive assistance from a machine than from human caregivers.

Interview 8: but for me it's easier to trust a machine that works in an automatic way that never varies. It's like an electronic and electric device, the help that they can provide cannot be different from that for which it was designed for. So for me, it's as reliable as a wall or a chair, as reliable as an object that is! ( $F$, 67 years-old, older person with RM)

Hence, older people expected that mobility assistance would be regular and precise, as opposed to the help provided by human caregivers, described as less reliable. This is an interesting finding as it suggests that older people with RM experience negativity with relation to their dependency on their caregivers. Indeed, traditional AT devices such as wheelchairs, were described here as passive, thus, less attractive than LLE:

Interview 11: Precisely, I see that as an active help. To me, that is neither being dependent on a machine nor being dominated by it. Not at all. It's the opposite, as it's me who dominates the machine to use it and do whatever I want. In my idea, it's always me who is in command of the machine, depending on my desire. I don't let myself get dominated by the machine. $(F, 62$ years-old, older person with RM)

Here, older people pictured themselves as active agents regarding AT and exoskeleton-use. LLE were considered like tools or aids that they would be able to command and control depending to their will and needs, not the other way around.

According to the second imaginary, our analysis identified more reluctance towards LLE. Exoskeletons were perceived as devices with the power to erase the humanity of end-users and even their minds. LLE were labelled 'robots':

Interview 10: She is swallowed by the machine and you cannot even see her anymore [pointing at the person wearing an exoskeleton in one of the images] I would have the feeling of becoming a robot with all that paraphernalia! She is totally dominated by the machine, and it's not a real person anymore, who is master of her own actions. $(F, 75$ years-old, older person with $R M)$

This second perspective was underpinned by the positive value of being assisted by human caregivers instead of 'machines'. Help in this context was signified as an opportunity to interact socially:

Interview 10: Frankly, from the moment help is required, I'd rather have people's help. They can bring something else, conversations and so on. Because even though the machine could help me to move around, it's only a physical help, while older people, often, well they are looking for that but also for company. No truly, I prefer being helped by a real person and not by a machine. $(F, 75$ years-old, older person with $R M$ )

This second imaginary was associated with the concern of being dominated by the machine. Agency here was highly valued, yet in a different way than the first imaginary:

Interview 5: I would be afraid to delegate such an important thing as my own walk to a machine. (M, 64 years-old, older person with RM)

In summary, two main contrasting imaginaries were identified in this third theme among older people with RM' accounts. Such imaginaries refer to views on the human-machine interaction embedded within meanings of the care relationship and the value of human agency.

Below, we synthetise main findings on older people's perspective (Table 1).

Table 1 Main themes capturing older people with RM's perspectives on LLE acceptance for everyday use

(a) Autonomy in everyday life can be enhanced through LLE, yet its acceptance requires learning

Feeling more independent from caregivers

Feeling less of a burden

Feeling less tired

vs

Integrating LLE to everyday life needs to be gradual and requires learning

(b) Contrasting views of the ageing process

Ageing is a 'natural' process that must not be countered vs

Ageing is a process that needs to be countered

(c) Contrasting imaginaries of the human-machine interaction

LLE as strongly reliable machines

It is easy to receive help from a machine

vs

LLE as machines are less reliable than humans

It is difficult to receive help from a machine 


\subsection{Informal Caregivers' Perspectives}

Overall, informal caregivers perceived the use of LLE among older people with RM as highly beneficial. Two main themes captured this perspective:

(a) LLE have the potential to relieve some of the burden related to care responsibilities and,

(b) the acceptance of LLE for everyday life use would require learning

\subsubsection{LLE have the Potential to Relieve Some of the Burden Related to Care Responsibilities}

According to informal caregivers, LLE had the potential to alleviate some of the burden of care responsibilities in the day-to-day. The emotional bond that participants described in relation to older people was particularly strong. Such a bond, however, could become burdensome and care activities were experienced as obligations.

Focus group 1: There is this dimension where, at times, I think that it's not at all tiring to go and visit her, but when it's over, I feel drained, since I play the moderator/to cheer her up, and then I try to spice up her life because... she expects me to come visit. $(F, 65$ years-old, informal caregiver at a residence)

From this perspective, taking care of older people with RM implied a taxing daily commitment. The great majority of participants explained the difficulty of their role, which they had to learn 'by doing', with little or no formal training. In this sense, the daily care of older people with RM could lead to situations that were perceived as too complex for them to handle. Hence, LLE were portrayed as helpful tools:

Focus group 1: I think that the exoskeleton would be maybe not really for her and her independence, but most of all for the person by her side, for instance, to me. I would appreciate that help! It's true that assistive means as this machine would be helpful for those who are side by side [with the older people with RM], like myself. (M, 75 years-old, informal caregiver at a residence)

This finding of informal caregivers' views strongly echoes with older people with RM's perspectives, according to which they also felt like a 'burden' to others and believed that LLE represented a solution to alleviate this burden.
In addition, LLE acceptance was enhanced by the perception that these tools would help maintain older people's mobility abilities, including: the strengthening and maintenance of muscular mass; improving body balance; beneficial effects due to the upright position and the decrease in psychological and mental fatigue needed to anticipate each movement.

Focus group 1: He would feel support provided by the machine [showing the exoskeleton in one of the pictures]. That tool would allow him to worry less about every movement to make, so he would feel less tired. $(F, 65$ years-old, informal caregiver at a residence)

Again, this finding echoes with older people with RM's perspectives. Moreover, informal caregivers perceived that LLE use would help improve older people's subjective wellbeing by participating to everyday life activities, beyond functional advantages of the exoskeleton:

Focus group 1: And if the person is able to find the mechanical ability with this assistance, maybe it could also help to achieve a better quality of life at the physical level, thanks to an increase in mobility, but also psychologically, to find joy again. (M, 65 years-old, Informal caregiver at a residence)

Informal caregivers argued that these benefits could be enhanced by the specific design of AUTONOMYO, enabling for the user to initiate her/his own walking movement. This represented a key quality to enhance the feeling of control over the device, instead of the opposite.

Focus group 2: It [the exoskeleton] enables a mechanical walk but the person induces the gesture, so she feels as the warden of the apparatus. It is her who is in command of the movement, so it's a great aid that makes the person feel worthy. She has really the impression of managing her own movement, let's put it like that. She is not captive or passive of the device. $(F, 73$ years-old, informal caregiver at a residence)

Such finding also resonates with certain views identified across older people accounts.

To summarise, this theme of informal caregivers' perspectives showed that LLE were largely beneficial 'tools' that could significantly alleviate their care duties, and furthermore help older people with RM through an increase in physical movement, and therefore, everyday life social activities, contributing to an embodied personal and social wellbeing. 


\subsubsection{The Acceptance of LLE for Everyday Life Use Would Require Ample Learning}

In line with older people with RM's accounts, informal caregivers perceived LLE acceptance as a learning process. According to participants, exoskeletons would need to be introduced gradually, as if they assumed that older people with RM would first reject the tool before adopting it, except for situations where AT such as wheelchairs or canes were already part of older people's everyday lives.

Focus group 2: The wheelchair and anything that helps in an ergonomic way, that she [older people with RM] accepts quite easily. At home, we have tools to help her climb the stairs, we have wheelchairs, we have walkers, canes. So, I think that she wouldn't have any trouble with it [the exoskeleton] ( $F, 26$ years-old, informal caregiver at a residence)

All informal caregivers agreed that older people with RM would adopt exoskeletons under the condition of feeling in control of the device. This finding was underpinned by the widely shared perception that LLE would relieve the felt burden of care.

In summary, this theme extended informal caregivers' shared perceptions of exoskeletons as promising solutions to relieve care duties by representing a tool that would allow older people with RM to move around in everyday life environments that have not yet been adapted for people with mobility impairments. Although such devices would need a gradual introduction, LLE would be integrated successfully to older people's daily lives.

Here, we summarise the main findings on psychosocial aspects on LLE acceptance produced by informal caregivers' accounts (Table 2).

\subsection{Professional Caregivers' Perspectives}

Professional caregivers accounts showed a certain degree of resistance towards the acceptance of LLE for everyday use. While participants acknowledged psychosocial benefits for older people with RM, they perceived LLE as a threat to the care relationship in healthcare settings.

We identified two main themes capturing the formal caregivers' perspective:

(a) LLE use would lead to a dehumanisation of the care relationship, and

(b) LLE and AT represent a potential threat for certain healthcare professions.

\subsubsection{LLE Use Would Lead to a Dehumanisation of the Care Relationship}

This theme described the fear perceived among professional caregivers towards LLE, as these devices were viewed as a threat to the care relationship, especially in the "care-athome' context. Their accounts stressed the major role that the human relationship dimension plays in their job. In this view, older people with RM would feel isolated by gaining autonomy through LLE use:

Focus group 3: As I was saying at first, they [older people with RM] will think that we will pass by less often and that they will be lonelier in solitude. So, they won't accept this device [pointing at an image representing an exoskeleton] because then we would pay less visits to their homes. Actually, it's neither the care nor the autonomy that they are looking for, but the company. This device would scarcely solve any problems. $(F, 45$ years-old, professional caregiver at a residence)
Table 2 Main themes capturing informal caregivers' perspectives on LLE acceptance for everyday use among older people with RM (a) LLE have the potential to relieve some of the burden related to care responsibilities

The emotional bond that links informal caregivers to older people with RM would become stronger given LLE use in everyday life contexts Integrating LLE to everyday life would improve wellbeing among older people with RM

(b) The acceptance of LLE for everyday life use would imply an extensive learning process

LLE acceptance among older people with RM constitutes a process that requires ample learning Eventually, LLE acceptance among older people with RM will be successful 
Professional caregivers perceived the functional benefits of LLE but feared the loss of important social interactions. To them, the human contact should not be replaced by LLE, nor any AT:

Focus group 3: We represent their [older people with RM] contact with the outer world. Thus, with this machine [pointing at an exoskeleton in one of the images] they would say 'well, they [professional caregivers] won't have a reason to pass by anymore'. They would see the machine [the exoskeleton] as a physical help, but they would lose the [human] presence. ( $F$, 52 years-old, professional caregiver at a residence)

To summarise this theme, professional caregivers were sceptical of LLE. The potential psychosocial benefits of this tool for older people were reduced to purely functional aspects.

\subsubsection{LLE and AT Represent a Threat for Certain Healthcare Professions}

Professional caregivers perceived a limited potential of LLE in healthcare settings, especially with older people with RM. Exoskeletons could only be seen as working tools, for example with the purpose of supporting a rehabilitation process rather than for everyday life use.

Focus group 3: That's why I'd rather see it [the exoskeleton] as a form of re-education. Yes, a little bit like physiotherapy where, twice a week, we go for a walk with that thing. ( $F, 52$ years-old, professional caregiver at a residence)

All professional caregivers, were reluctant towards LLE and highlighted potential risks:

Focus group 4: If that [the exoskeleton] falls on the user, it's really dangerous. It's imperative in this context to prevent falling [among the older people] because if they fall with this thing, it's even more dangerous that without wearing any device ( $F, 35$ yearsold, professional caregiver at a residence)

This second theme suggests that professional caregivers perceived LLE and AT as a potential threat to their job. The thought of fearing to be replaced in the future by AT seemed unbearable:

Focus group 3: So eventually, with this machine [exoskeletons] aren't we endangering our jobs even more? in the sense that people will be needing less and less our services and that they will call for these services increasingly less? And that everything will be replaced by machines and automatic things precisely. It is scary if we think like that because our job is maybe in danger, as the result of replacing everything by devices or other technological functions! ( $F, 52$ years old, informal caregiver at a residence)

Such a threat was enhanced by a broader concern: the extra workload that was attributed to the increased and rapid digitalization of healthcare. LLE were viewed as a burden rather than an aid to their work. In this sense, technological devices were referred to as mere gadgets rather than proper tools within the context of their profession:

Focus group 4: The main problem of working in residencies for older people relies on the heavy workload, which we refer to as 'the electro-numeric workload'. This means that each year, they [the top managerial levels] keep bringing special walkers, special alarms, and so on. (M, 56 years-old, professional caregiver at a residence)

In summary, professional caregivers showed a certain degree of resistance towards LLE use among older people with RM in healthcare settings. This was underpinned by a broader fear of the rapid expansion of AT in healthcare contexts (Table 3).

To conclude the findings section, we present a table that synthetises main outcomes around two crosscutting psychosocial considerations-'perceptions of the ageing process' and 'perceptions on the human-machine interaction'-based on each population's perspective (Table 4).

The first psychosocial consideration regards specific perceptions of the ageing process. Among older people with RM, two perspectives were identified: those accepting the process of ageing, defined by a 'natural' physical mobility loss, and those who were willing to combat the mobility

Table 3 Main themes capturing professional caregivers' perspectives on LLE acceptance for everyday use among older people with RM: nursing homes and 'care at home' contexts

\section{(a) LLE use would lead to a dehumanisation of the care relationship}

The social bond that links professional caregivers to older people with RM would be threatened if LLE were used by older people in everyday life contexts

In healthcare settings, integrating LLE to everyday life would decrease wellbeing among older people with RM

(b) LLE and AT represent a threat for healthcare professionals

In healthcare settings, the use of LLE by older people with RM would be useful for rehabilitation purposes only LLE and AT as a threat to healthcare jobs 
Table 4 Synthesis of psychosocial considerations on LLE acceptance for everyday life use among older people with RM

\begin{tabular}{|c|c|c|c|}
\hline Social actors concerned & $\begin{array}{l}\text { User prox- } \\
\text { imity with } \\
\text { LLE }\end{array}$ & Perceptions of the ageing process & Perceptions on the human-machine interaction \\
\hline Older people with RM & Primary & $\begin{array}{l}\text { Ageing as main factor to adopt the LLE, yet LLE } \\
\text { use requires an ample learning process }\end{array}$ & $\begin{array}{l}\text { LLE enhances autonomy } \\
\text { LLE are a potential relief to feeling like a burden } \\
\text { to others } \\
\text { Trust in technology is a main factor to adopt LLE }\end{array}$ \\
\hline Informal caregivers & Secondary & $\begin{array}{l}\text { LLE use requires a learning process in order to be } \\
\text { used by older people }\end{array}$ & $\begin{array}{l}\text { LLE enhances autonomy } \\
\text { LLE are an undeniable relief for the care relation- } \\
\text { ship } \\
\text { Mostly trust the technology }\end{array}$ \\
\hline Professional caregivers & Secondary & $\begin{array}{l}\text { LLE may de-humanize the care relationship, } \\
\text { which is central to older people's wellbeing }\end{array}$ & $\begin{array}{l}\text { LLE are an economic threat to healthcare jobs } \\
\text { Resistance towards LLE and technology are linked } \\
\text { to broader fears on the digitalization of health- } \\
\text { care }\end{array}$ \\
\hline
\end{tabular}

changes due to ageing through LLE use. Informal caregivers' visions of ageing implied the eventual LLE use by older people with RM even though the learning process would take time. Professional caregivers perceived LLE use as a threat to the care relationship, underpinned by the perception of ageing as needing professional care based in human interactions.

The second psychosocial consideration refers to the human-machine interactions. For older people with RM and informal caregivers, LLE use was associated to enhanced autonomy in everyday life, as well as a relief of the burden experienced within the care relationship. At the same time, older people with RM positioned themselves in opposing ways, trusting the machine yet fearing that the latter could take over their personal agency. Finally, professional caregivers perceived the human-machine interaction as an economic threat-perhaps linked to a fear of losing their jobs due to LLE. Indeed, such a threat seemed more broadly underpinned by concerns of the rapid digitalisation of healthcare.

\section{Synthesis}

This exploratory study aimed at exploring perceptions regarding potential Lower Limb Exoskeletons (LLE) for everyday use among an older population with reduced mobility (RM). Three populations that could particularly benefit from this technological advance were included: older people with RM and their close entourage-informal and professional caregivers. To do so, we implemented an interdisciplinary study between robotics engineers and health psychologists. We adopted a qualitative approach of health psychology using AUTONOMYO, a LLE prototype produced by the robotics engineers. Our study addressed meanings attached to experiences, necessary for a better understanding of LLE acceptance. Our qualitative methodology was underpinned by a grounded theory framework, aimed at exploring participants' views by focusing on their accounts, focusing on meanings. Accounts were stimulated by images presented of AUTONOMYO worn by an older person across different everyday life contexts (shopping, walking, social interactions, etc.). Each of the three perspectives was considered in the qualitative analysis (thematic analysis). This grounded theory approach was complementary to previous recent work analysing Assistive Technology (AT) acceptance and, more particularly, LLE use among senior adults based on more deductive models (e.g. STAM) $[11,15,16]$.

Main findings highlighted key crosscutting psychosocial considerations linked to end-users' perceptions regarding LLE acceptance alongside the widely positive view that LLE could enhance autonomy: the need to consider subjective perceptions of the ageing process when studying LLE acceptance among older people with RM, and the importance of analysing perceptions on the human-machine interaction.

\section{Discussion and Conclusion}

In the present study, LLE were perceived as promising solutions for older people with RM, especially among older people with RM and informal caregivers. Such devices were defined as potentially useful tools. This finding builds upon previous recent studies in the field within Western contexts, namely Europe $[16,47]$. Participants strongly believed that LLE could address experienced difficulties resulting from the lack of autonomy due to ageing. Older people with $\mathrm{RM}$ and informal caregivers described the burden they 
experienced regarding the strong dependency of the older person on the caregiver in the care relationship due to mobility impairments. Both populations had high hopes to use LLE to help relieve this psychosocial burden. This finding contributes to the current literature, namely on European attitudes towards assistive robots, by pointing out potential psychosocial benefits perceived by young olds regarding AT, such as LLE. Indeed, younger generations of older adults seem less reluctant than people aged over 75 [48].

Our outcomes highlighted the key role of perceptions of the ageing process among each population considered in our study. Among the group of older people with RM, two different perceptions of ageing were analysed: ageing as a 'natural' process that does not need to be prevented and a second one where it can be combatted via AT, such as LLE. Depending on whether the process of growing old was signified as needing to be prevented or, on the contrary, accepted, LLE were viewed more or less positively. The acceptance of exoskeletons was perceived by older people with RM and informal caregivers as a progressive phenomenon that required ample learning. In both cases, LLE were believed to eventually be part of their daily lives.

LLE were perceived by all three populations as implying a specific 'human-machine' relationship, fundamentally different from human-human interactions. Beyond this common psychosocial consideration, each population had a particular vision:

Both older people with RM and informal caregivers' accounts revealed trust towards exoskeletons, perceived as machines that were more reliable than human assistance. This assertion was enhanced by the strong hope attached to exoskeletons, seen as a solution to ease the burden of mobility impairments for both populations. The feeling of trust towards exoskeletons was referred to by older people with RM when they presented themselves as agents who would be able to control the device. Certain fears arose when the device was perceived as a machine that could dominate the user with scarce control over it. This finding seemed closely linked to the value attached to 'agency' in individualised Western societies [49]. Indeed, technology, via the human-machine interaction, shall be regarded as a tool that is used at the service of people's health and wellbeing, respecting subjective needs and specific contexts of use.

Professional caregivers positioned themselves as more resistant to LLE acceptance with regard to the human-machine interaction. This technology was not perceived as appropriate for therapeutic use among older people with RM. From this standpoint, older people with RM were seen in need of care that only human beings could provide. LLE were portrayed more broadly, as threatening the care relationship. This feeling of menace seemed underpinned by a broader fear of being replaced by the machine and, thus, losing their jobs. This perception was related to a sense of resistance expressed towards the rapid and massive digitalization of healthcare, and LLE were perceived as part of this shift. This finding could be related to the fact that professional caregivers' care relationship carries an economic dimension linked to the job, as opposed to that characterising informal caregivers, defined as more emotional which can often be burdensome. Also, Western societies seem more conservative with regard to technological devices compared to other societies such as the Japanese culture [50-52].

Though the present exploratory study contributes to the understanding of the psychosocial factors at play, it had its limitations, namely the specificity of the sample-limited to the French Swiss sociocultural context. Also, due to ethical and security reasons, we were constrained to use photo-elucidation as stimuli, instead of inviting participants to wear the LLE which would have provided them with a more ideal experience. As AUTONOMYO was still a prototype at the time of the study there were clear restrictions to its use. Despite these limitations, the interdisciplinary collaboration using a qualitative approach in health psychology was particularly fruitful to move beyond functional considerations on acceptance underlined by recent literature [48, 53], and provide psychosocial insight of LLE acceptance for everyday use in the context of gerontology. In this sense, our approach enabled us to further understand end-users' perspectives in inductive ways.

In contemporary times, ageing has become a major societal challenge in terms of health costs while, paradoxically, technology has never been more present in the fields of health and gerontology. As researchers, we must reflect on our collective responsibility to define how to contribute to improve older peoples' quality of life in their day-to-day, by putting technology, such as LLE, at their service. Beyond senior adult populations, their entourage is at the heart of the challenges faced by older people's mobility impairments in everyday life, hence they also need to be included in this reflection [54]. Qualitative research has the potential to investigate people's views on technology use and has shown to be reliable $[55,56]$. This form of knowledge is complementary to those produced using experimental approaches. Promising future perspectives could thus involve the use of mixed-methods research designs able to articulate qualitative and quantitative data. Such designs would be highly useful to confirm and develop the two main psychosocial considerations outlined in the present research.

Funding Open Access funding provided by Université de Lausanne. Programme Collaborative Research on Science and Society - CROSS UNIL-EPFL (26046003). Project funded between January 2019 and June 2021. 
Data Availability The data that support the findings of this study are available on request from the corresponding author. The data are not publicly available due to their containing information that could compromise the privacy of research participants.

Code Availability Not applicable.

\section{Declarations}

Conflict of interest The authors declare that they have no conflict of interest.

Open Access This article is licensed under a Creative Commons Attribution 4.0 International License, which permits use, sharing, adaptation, distribution and reproduction in any medium or format, as long as you give appropriate credit to the original author(s) and the source, provide a link to the Creative Commons licence, and indicate if changes were made. The images or other third party material in this article are included in the article's Creative Commons licence, unless indicated otherwise in a credit line to the material. If material is not included in the article's Creative Commons licence and your intended use is not permitted by statutory regulation or exceeds the permitted use, you will need to obtain permission directly from the copyright holder. To view a copy of this licence, visit http://creativecommons.org/licenses/by/4.0/.

\section{References}

1. Bülow MH, Söderqvist T (2014) Successful ageing: a historical overview and critical analysis of a successful concept. J Aging Stud 31:139-149. https://doi.org/10.1016/j.jaging.2014.08.009

2. Erikson EH, Erikson JM, Kivnick HQ (1994) Vital involvement in old age. WW Norton \& Company

3. Jamieson A, Harper S, Victor C (1997) Critical approaches to ageing and later life. McGraw-Hill Education

4. Katz S (2019) Cultural aging: life course, lifestyle, and senior worlds. University of Toronto Press

5. Turner BS (1996) For Weber: essays on the sociology of fate. Sage

6. Santiago-Delefosse M (2014) The need for an 'Embodied-Societal-Psycholohical' (ESP) model of illness experience. In: Murray M (ed) Critical health psychology, 2nd edn. Macmillan International Higher Education, pp 36-55

7. Lutz W, Sanderson W, Scherbov S (2008) The coming acceleration of global population ageing. Nature 451:716-719. https://doi. org/10.1038/nature06516

8. United Nations (2019) World population prospects 2019:highlights. United Nations

9. Dogra S, Stathokostas L (2012) Sedentary behavior and physical activity are independent predictors of successful aging in middle-aged and older adults. J Aging Res 2012:8. https://doi.org/10. 1155/2012/190654

10. Seshadri S, Wolf PA (2007) Lifetime risk of stroke and dementia: current concepts, and estimates from the Framingham Study. Lancet Neurol 6:1106-1114. https://doi.org/10.1016/S1474-4422(07) 70291-0

11. Hill D, Holloway CS, Ramirez DZM et al (2017) What are user perspectives of exoskeleton technology? A literature review. Int J Technol Assess Health Care 33:160-167. https://doi.org/10.1017/ S0266462317000460

12. Ortlieb A, Bouri M, Baud R, Bleuler H (2017) An assistive lower limb exoskeleton for people with neurological gait disorders. In:
2017 international conference on rehabilitation robotics (ICORR). IEEE, pp 441-446

13. Ortlieb A, Bouri M, Bleuler H (2017) AUTONOMYO: design challenges of lower limb assistive device for elderly people, multiple sclerosis and neuromuscular diseases. In: Wearable robotics: challenges and trends. Springer International Publishing, pp 439-443

14. Grimmer M, Riener R, Walsh CJ, Seyfarth A (2019) Mobility related physical and functional losses due to aging and disease- a motivation for lower limb exoskeletons. J NeuroEng Rehabil 16:2. https://doi.org/10.1186/s12984-018-0458-8

15. Shore L, Power V, de Eyto A, O'Sullivan L (2018) Technology acceptance and user-centred design of assistive exoskeletons for older adults: a commentary. Robotics 7:3. https://doi.org/10.3390/ robotics 7010003

16. Shore L, de Eyto A, O'Sullivan L (2020) Technology acceptance and perceptions of robotic assistive devices by older adultsimplications for exoskeleton design. Disabil Rehabil Assist Technol. https://doi.org/10.1080/17483107.2020.1817988

17. Santiago-Delefosse M, del Rio Carral M (2017) Manières de voir le monde en sciences sociales et humaines et en psychologie. In: Les méthodes qualitatives en psychologie et sciences humaines de la santé. Dunod

18. Lyons AC, Chamberlain K (2017) Critical health psychology. In: Gough B (ed) The Palgrave handbook of critical social psychology. Palgrave, pp 533-555

19. Chamberlain K, Lyons A (2020) Critical and qualitative approaches to behavior change. In: Hagger MS, Cameron L, Hamilton $\mathrm{K}$ et al (eds) The handbook of behavior change. Cambridge University Press, Cambridge

20. Lyons AC, Chamberlain K (2006) Health psychology: a critical introduction. Cambridge University Press

21. Robinson H, MacDonald B, Broadbent E (2014) The role of healthcare robots for older people at home: a review. Int J Soc Robot 6:575-591. https://doi.org/10.1007/s12369-014-0242-2

22. Miller DP (1998) Assistive robotics: an overview. In: Mittal $\mathrm{VO}$ et al (eds) Assistive technology and artificial intelligence. Springer, pp 126-136

23. Zhang L, Liu Y, Wang R et al (2021) Modeling and simulation of a human knee exoskeleton's assistive strategies and interaction. Front Neurorobotics. https://doi.org/10.3389/fnbot.2021.620928

24. Zhang L, Li Z, Hu Y et al (2021) Ankle joint torque estimation using an EMG-driven neuromusculoskeletal model and an artificial neural network model. IEEE Trans Autom Sci Eng 18:564573. https://doi.org/10.1109/TASE.2020.3033664

25. Lee H, Lee S, Chang WH et al (2017) A wearable hip assist robot can improve gait function and cardiopulmonary metabolic efficiency in elderly adults. IEEE Trans Neural Syst Rehabil Eng 25:1549-1557. https://doi.org/10.1109/TNSRE.2017.2664801

26. Hartigan C, Kandilakis C, Dalley S et al (2015) Mobility outcomes following five training sessions with a powered exoskeleton. Top Spinal Cord Inj Rehabil 21:93-99. https://doi.org/10. 1310/sci2102-93

27. Louie DR, Eng JJ, Lam T, Spinal Cord Injury Research Evidence (2015) Gait speed using powered robotic exoskeletons after spinal cord injury: a systematic review and correlational study. J NeuroEng Rehabil 12:82. https://doi.org/10.1186/s12984-015-0074-9

28. Kawamoto H, Lee S, Kanbe S, Sankai Y (2003) Power assist method for HAL-3 using EMG-based feedback controller. In: SMC'03 conference proceedings. 2003 IEEE international conference on systems, man and cybernetics. Conference themesystem security and assurance (Cat. No.03CH37483), vol 2, pp $1648-1653$ 
29. Kusuda Y (2009) In quest of mobility - Honda to develop walking assist devices. Ind Robot Int J 36:537-539. https://doi.org/10. 1108/01439910910994597

30. Verrusio W, Gianturco V, Cacciafesta M et al (2017) Fall prevention in the young old using an exoskeleton human body posturizer: a randomized controlled trial. Aging Clin Exp Res 29:207-214. https://doi.org/10.1007/s40520-016-0540-7

31. Gramstad A, Storli SL, Hamran T (2014) Older individuals' experiences during the assistive technology device service delivery process. Scand J Occup Ther 21:305-312. https://doi.org/10.3109/ 11038128.2013.877070

32. Laitinen A, Niemelä M, Pirhonen J (2016) Social robotics, elderly care, and human dignity: a recognition-theoretical approach. In: Can WSR, Seibt SDJ (eds) What social robots can and should do: proceedings of robophilosophy. IOS Press, pp 155-163

33. Papadimitriou C (2016) To move as a human: comment on" The embodiment of assistive devices-from wheelchair to exoskeleton" by M. Pazzaglia and M. Molinari. Phys Life Rev 16:186-187. https://doi.org/10.1016/j.plrev.2016.01.022

34. Pazzaglia M, Molinari M (2016) The embodiment of assistive devices-from wheelchair to exoskeleton. Phys Life Rev 16:163175. https://doi.org/10.1016/j.plrev.2015.11.006

35. Huo W, Mohammed S, Amirat Y, Kong K (2016) Active impedance control of a lower limb exoskeleton to assist sit-to-stand movement. In: 2016 IEEE international conference on robotics and automation (ICRA), pp 3530-3536

36. Glaser BG, Strauss AL (1967) The discovery of grounded theory. Weidenfield \& Nicolson

37. Charmaz K, Henwood K (2013) Grounded theory methods for qualitative psychology. In: Willig C, Rogers WS (eds) The SAGE handbook of qualitative research in psychology. Sage Publications, pp 238-257

38. Cromby J (2007) Toward a psychology of feeling. Int J Crit Psychol 21:118

39. Radley A (1993) The world of illness. Routledge

40. Cromby J (2011) The greatest gift? Happiness, governance and psychology. Soc Personal Psychol Compass 5:840-852. https:// doi.org/10.1111/j.1751-9004.2011.00398.x

41. Guba EG, Lincoln YS (1994) Competing paradigms in qualitative research. Handbook of qualitative research. Sage Publications Inc, pp 105-117

42. Blanchet A (2007) L'enquête et ses méthodes: l'entretien. Armand Colin

43. Braun V, Clarke V (2013) Successful qualitative research: a practical guide for beginners. Sage

44. Harper D (2002) Talking about pictures: a case for photo elicitation. Vis Stud 17:13-26. https://doi.org/10.1080/1472586022 0137345
45. Braun V, Clarke V (2006) Using thematic analysis in psychology. Qual Res Psychol 3:77-101. https://doi.org/10.1191/1478088706 qp063oa

46. Braun V, Clarke V (2019) Reflecting on reflexive thematic analysis. Qual Res Sport Exerc Health 11:589-597. https://doi.org/10. 1080/2159676X.2019.1628806

47. Jung MM, Ludden GDS (2018) Potential of exoskeleton technology to assist older adults with daily living. In: Extended abstracts of the $2018 \mathrm{CHI}$ conference on human factors in computing systems. Association for Computing Machinery, New York, NY, USA, pp 1-6

48. Hoppe JA, Johansson-Pajala R-M, Gustafsson C, et al (2020) Assistive robots in care: Expectations and pereceptions of older people. In: Aging between participation and simulation. De Gruyter Open, pp 139-156

49. del Rio CM, Lyons A (2020) Embodying health behaviours in everyday life: the social and gendered practices of female senior managers. Psychol Health. https://doi.org/10.1080/08870446. 2020.1743292

50. Kitano N (2006) A comparative analysis: social acceptance of robots between the West and Japan

51. Kaplan F (2004) Who is afraid of the humanoid? Investigating cultural differences in the acceptance of robots. Int J Humanoid Robot 01:465-480. https://doi.org/10.1142/S0219843604000289

52. Kitano N (2006) 'Rinri': an incitement towards the existence of robots in Japanese society. Int Rev Inf Ethics 6:78-83

53. Shore L, Power V, Hartigan B et al (2020) Exoscore: a design tool to evaluate factors associated with technology acceptance of soft lower limb exosuits by older adults. Hum Factors J Hum Factors Ergon Soc 62:391-410. https://doi.org/10.1177/0018720819 868122

54. Power V, de Eyto A, Bauer C et al (2018) Exploring user requirements for a lower body soft exoskeleton to assist mobility. In: Bai S, Virk GS, Sugar TS (eds) Wearable exoskeleton systems: design, control and applications. Institution of Engineering and Technology, pp 67-95

55. Morse JM, Barrett M, Mayan M et al (2002) Verification strategies for establishing reliability and validity in qualitative research. Int J Qual Methods 1:13-22. https://doi.org/10.1177/1609406902 00100202

56. Sousa D (2014) Validation in qualitative research: general aspects and specificities of the descriptive phenomenological method. Qual Res Psychol 11:211-227. https://doi.org/10.1080/14780887. 2013.853855

Publisher's Note Springer Nature remains neutral with regard to jurisdictional claims in published maps and institutional affiliations. 